\title{
Resonant atom Majorana mixing
}

\section{J. Bernabeu* and A. Segarra}

Department of Theoretical Physics, University of Valencia and IFIC, Joint Centre UV-CSIC

E-46100 Burjassot, Valencia

E-mail: Jose.Bernabeu@uv.es, Alejandro.Segarra@uv.es

\begin{abstract}
Massive neutrinos demand to ask whether they are Dirac or Majorana particles. Majorana neutrinos are an irrefutable proof of physics beyond the Standard Model. Neutrinoless double electron capture is not a process but a virtual $\Delta L=2$ mixing between a parent ${ }^{A} Z$ atom and a daughter ${ }^{A}(Z-2)$ excited atom with two electron holes. As a mixing between two neutral atoms and the observable signal in terms of emitted two-hole X-rays, the strategy, experimental signature and background are different from neutrinoless double beta decay. The mixing is resonantly enhanced for almost degeneracy and, under these conditions, there is no irreducible background from the standard two-neutrino channel. We reconstruct the natural time history of a nominally stable parent atom since its production either by nature or in the laboratory. After the time periods of atom oscillations and the decay of the short-lived daughter atom, at observable times the relevant "stationary" states are the mixed metastable long-lived state and the short-lived excited state, as well as the ground state of the daughter atom. Their natural population inversion is most appropriate for exploiting the bosonic nature of the observed X-rays by means of stimulating X-ray beams. Among different observables of the atom Majorana mixing, we include the enhanced rate of stimulated X-ray emission from the long-lived metastable state by a high-intensity X-ray beam. a gain factor of 100 can be envisaged at current XFEL facilities. On the other hand, the historical population of the daughter atom ground state can be probed by exciting it with a current pulsed optical laser, showing the characteristic absorption lines: the whole population can be excited in a shorter time than typical pulse duration.
\end{abstract}

Corfu Summer Institute 2017 'School and Workshops on Elementary Particle Physics and Gravity' 2-28 September 2017

Corfu, Greece

\footnotetext{
* Speaker.
} 


\section{Massive neutrinos}

Neutrino flavour oscillations have been observed in atmospheric [1], solar [2], reactor [3] and accelerator [4] sectors. They imply that neutrinos have a mass and the flavour neutrinos are mixtures of the neutrinos with definite mass. Up to now two mass differences $\Delta m_{21}^{2},\left|\Delta m_{31}^{2}\right|$ and three mixings have already been measured.

Among the open questions in neutrino physics, the most important problem is whether neutrinos are either Dirac, with a conserved global lepton number, or Majorana, truly neutral, particles. Starting from the observed active chiral neutrino field $v_{L}$, responsible of weak interactions, one can build a Dirac mass term

$$
\bar{v}_{R} m_{D} v_{L}
$$

with its chiral mirror partner $v_{R}$, which is sterile for all gauge interactions. A term like this conserves global lepton number and can be originated by means of the standard Higgs sector. The two degenerate definite masses are associated to neutrinos and antineutrinos in the complex field with a combination of these two chiralities. As for any other Dirac particle, we have four degrees of freedom $v_{L}, v_{R}, v_{L}^{c}, v_{R}^{c}$.

However, a priori the chirality flip needed to build a mass term can also be obtained with the already observed interacting antineutrino $v_{L}^{c}$ by means of a Majorana mass term

$$
\bar{v}_{L}^{c} m_{M} v_{L}
$$

Evidently this mass term breaks all charges by two units and, as a consequence, it is forbidden for quarks and charged leptons with exact conservation of colour and electric charges. For neutrinos, it is a $\Delta L=2$ term. Such a term cannot be originated by spontaneous gauge symmetry breaking from a renormalizable dimension 4 operator with the standard Higgs sector, and it needs a dimension 5 operator in the effective gauge symmetric Lagrangian. Whereas this origin provides a rationale for the small neutrino masses, one needs a beyond-standard-model high energy scale. The field with definite mass is the truly neutral combination of $v_{L}$ and $v_{L}^{c}$. Majorana neutrinos have two degrees of freedom $v_{L}, v_{L}^{c}$.

For three families of Majorana neutrinos, the PMNS flavour mixing matrix contains two diagonal additional observable $\mathrm{CP}$ violating phases having their origin in the relative phase of neutrino and antineutrino components of the definite mass field. Their observable appearance can then be effective through mass effects only. The difficulty in distinguishing Dirac versus Majorana neutrinos is due to the smallness of mass terms versus available relativistic neutrino energies. The known way to study this problem is the experimental search of a $\Delta L=2$ transition like neutrinoless double $\beta$ decay $(0 v \beta \beta)$ of atomic nuclei

$$
{ }^{A} Z \rightarrow{ }^{A}(Z+2)+2 e^{-}
$$

with $Z$ the atomic number and $A$ the mass number. It proceeds through the diagram of Fig. 1.

As seen in this diagram, the process becomes possible with a Majorana mass insertion in the 


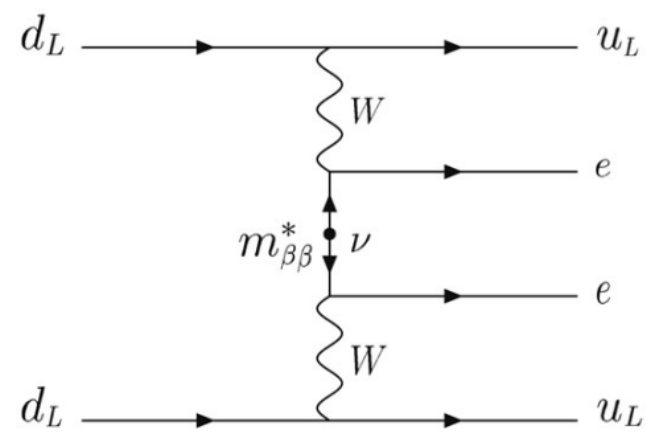

Figure 1: The amplitude for neutrinoless double $\beta$ decay.

virtual neutrino propagator. For three families the amplitude is then proportional to

$$
m_{\beta \beta} \equiv \sum_{i} U_{e i}^{2} m_{v_{i}}
$$

which is a coherent combination of the three neutrino masses.

This $\Delta L=2$ process exists only if neutrinos are Majorana. Its experimental signature is a peak $T_{e e}=Q$ in the summed electron energies at the $Q$-value of the atomic transition superposed to the continuum spectrum $T_{e e}<Q$ for the standard two-neutrino double beta decay $(2 v \beta \beta)$, which constitutes an irreducible background. Good energy resolution in the detector is then an experimental request. The present experimental situation, compared to the expected $m_{\beta \beta}$ (1.4), is given [5] in Fig. 2. Next generation experiments plan to reach the region of the inverted hierarchy in the neutrino mass spectrum $\Delta m_{31}^{2}<0$.

An alternative to $0 v \beta \beta$ is the transition induced by neutrinoless double electron capture (0vECEC)

$$
{ }^{A} Z+2 e^{-} \rightarrow{ }^{A}(Z-2)^{*}
$$

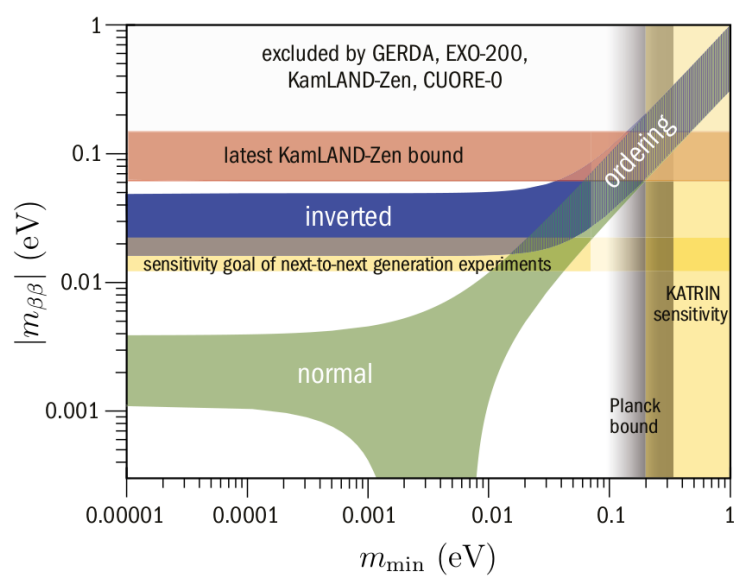

Figure 2: The expected $m_{\beta \beta}$ for Majorana neutrinos as function of the lowest neutrino mass, compared with present experimental limits. 


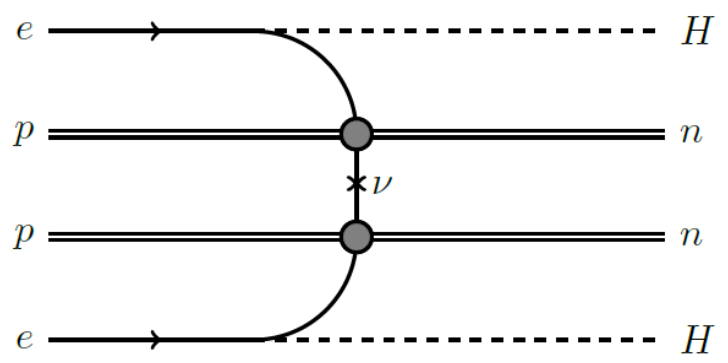

Figure 3: The amplitude for neutrinoless double electron capture.

which proceeds through the diagram of Fig. 3 with the capture of two atomic electrons by the nucleus leading to a final atom with two electron holes.

This transition is actually a $\Delta L=2$ virtual mixing between two states of different neutral atoms with the same mass number A, the daughter atom in a highly excited state with two electron holes in the inner shells. The amplitude $M_{21}$ for this Majorana mixing is proportional [6] in a good approximation to

$$
M_{21}=m_{\beta \beta}^{*}\left(\frac{G_{F} \cos \theta_{C}}{\sqrt{2}}\right)^{2}\left\langle F_{21}\right\rangle \frac{g_{A}^{2}}{2 \pi} M_{0 v}
$$

with the complex conjugate of $m_{\beta \beta}$ in Eq.(1.4) through the CP phases of the PMNS neutrino mixing matrix elements $U_{e i}$. Notice that this expression has three clearly differentiated factors, coming from particle, atomic and nuclear physics, respectively. $\left\langle F_{21}\right\rangle$ corresponds to the double electron wave functions in the nuclear volume and $M_{0 v}$ is the nuclear matrix element. State-of-the-art calculations of $M_{0 v}$ are available from nuclear QRPA [7] and IBM [8] approaches.

The virtual mixing, not conserving energy-momentum between parent and daughter atomic states, becomes observable through the signal of X-ray emission in the daughter atom to its ground state. The kinematics of the process is described in Fig. 4 with a self-explanatory notation.

As discussed later, the effect of the Majorana mixing $M_{21}$ is enhanced under the resonance condition $\Delta \sim \Gamma$. The $\Delta L=2$ mixing is induced by Majorana neutrinos only, the X-ray emission has the signature that its transition energy is $Q$ and there is no irreducible background from the

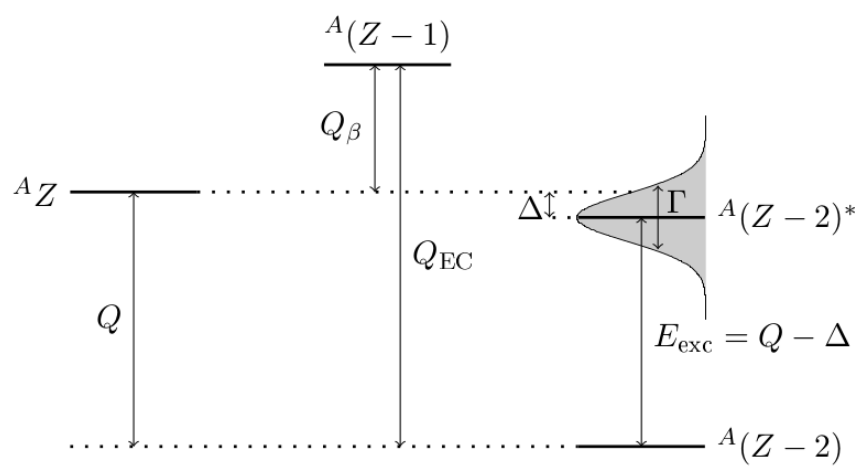

Figure 4: The kinematics for $X$-ray emission from the ground state of the parent ${ }^{A} Z$ atom to the ground state of the daughter ${ }^{A}(Z-2)$ atom. 
standard two-neutrino decay under the resonance condition.

In section 2 we describe the two-state effective Hamiltonian in the basis of the atomic states ${ }^{A} Z$ and ${ }^{A}(Z-2)^{*}$ and solve for the states of definite time evolution. The resonant enhancement of the mixing will become apparent. Section 3 reconstructs the natural time history of an initial parent atom ${ }^{A} Z$ with the objective of finding, at observable times, the current physical state and the relative populations of the three states which are involved. In section 4 both spontaneous observables and the idea of stimulating transitions for providing a signal of the $\Delta L=2$ atom mixing are discussed. Section 5 presents our conclusions.

\section{The evolution Hamiltonian}

In the basis of the $\left|{ }^{A} Z\right\rangle$ and $\left|{ }^{A}(Z-2)^{*}\right\rangle$ states, which we refer to as 1 and 2, the dynamics of this two-state system of interest is governed by the effective Hamiltionian

$$
\mathbf{H}=\mathbf{M}-\frac{i}{2} \boldsymbol{\Gamma}=\left[\begin{array}{cc}
M_{1} & M_{21}^{*} \\
M_{21} & M_{2}
\end{array}\right]-\frac{i}{2}\left[\begin{array}{ll}
0 & 0 \\
0 & \Gamma
\end{array}\right],
$$

with a Majorana $\Delta L=2$ mass mixing $M_{21}$ from Eq.(1.6). The anti-Hermitian part of this Hamiltonian is due to the instability of $\left|{ }^{A}(Z-2)^{*}\right\rangle$, which de-excites into $\left.\left.\right|^{A}(Z-2)_{\text {g.s. }}\right\rangle$, external to the two-body system in Eq.(2.1), emitting its two-hole characteristic X-ray spectrum.

Besides being non-Hermitian, $\mathbb{H}$ is not a normal operator, i.e. $[\mathbf{M}, \boldsymbol{\Gamma}] \neq 0$. As a consequence, $\mathbf{M}$ and $\boldsymbol{\Gamma}$ are not compatible. The states of definite time evolution, eigenstates of $\mathbb{H}$, have complex eigenvalues and are given in non-degenereate perturbation theory by

$$
\begin{array}{ll}
\left|\lambda_{L}\right\rangle=|1\rangle+\alpha|2\rangle, & \lambda_{L} \equiv E_{L}-\frac{i}{2} \Gamma_{L}=M_{1}+|\alpha|^{2}\left[\Delta-\frac{i}{2} \Gamma\right], \\
\left|\lambda_{S}\right\rangle=|2\rangle-\beta^{*}|1\rangle, & \lambda_{S} \equiv E_{S}-\frac{i}{2} \Gamma_{S}=M_{2}-\frac{i}{2} \Gamma-|\alpha|^{2}\left[\Delta-\frac{i}{2} \Gamma\right],
\end{array}
$$

with $\Delta=M_{1}-M_{2}$. Eq.(2.2) tells us that $\Gamma_{L, S}$ are not the eigenvalues of the $\mathbb{\Gamma}$ matrix. The eigenstates are modified to first order in $M_{21}$,

$$
\alpha=\frac{M_{21}}{\Delta+\frac{i}{2} \Gamma}, \quad \beta=\frac{M_{21}}{\Delta-\frac{i}{2} \Gamma},
$$

so the "stationary" states of the system do not have well-defined atomic properties: both the number of electrons and their atomic properties are a superposition of $Z$ and $Z-2$. Also, these states are not orthogonal-their overlap is given by

$$
\left\langle\lambda_{S} \mid \lambda_{L}\right\rangle=\alpha-\beta=-i \frac{M_{21} \Gamma}{\Delta^{2}+\frac{1}{4} \Gamma^{2}},
$$

with its non-vanishing value due to the joint presence of the mass mixing $M_{21}$ and the decay width $\Gamma$. Notice that $\operatorname{Im}\left(M_{21}\right)$ originates a real overlap. 
As seen in Eq.(2.2), the modifications in the corresponding eigenvalues appear at second order in $\left|M_{21}\right|$ and they are equidistant with opposite sign. Since these corrections are small, from now on we will use the values

$$
\begin{array}{ll}
E_{L} \approx M_{1}, & E_{S} \approx M_{2}, \\
\Gamma_{L} \approx|\alpha|^{2} \Gamma, & \Gamma_{S} \approx \Gamma .
\end{array}
$$

The only relevant correction at order $|\alpha|^{2}$ is the one to $\Gamma_{L}$, since $|1\rangle$ was a stable state-even if it is small, the mixing produces a non-zero decay width.

This result shows that, at leading order, the Majorana mixing becomes observable through $\Gamma_{L} \propto|\alpha|^{2}$. The value of $\alpha$ in Eq.(2.3) emphasizes the relevance of the condition $\Delta \sim \Gamma$, which produces a Resonant Enhancement [6] of the effect of the $\Delta L=2$ mass mixing $M_{21}$.

\section{Natural time history for initial ${ }^{A} Z$}

As seen in Eq.(2.2), the states $\left|{ }^{A} Z\right\rangle$ and $\left|{ }^{A}(Z-2)^{*}\right\rangle$ are not the stationary states of the system. For an initially prepared $\left|{ }^{A} Z\right\rangle$, the time history is far from trivial and the appropriate language to describe the system short times after is that of Atom Oscillations between $\left|{ }^{A} Z\right\rangle$ and $\left|{ }^{A}(Z-2)^{*}\right\rangle$ due to the interference of the amplitudes through $\left|\lambda_{S}\right\rangle$ and $\left|\lambda_{L}\right\rangle$ in the time evolution. The time-evolved $\left|{ }^{A} Z\right\rangle$ gives rise to the appearance probability

$$
\left|\left\langle{ }^{A}(Z-2)^{*} \mid{ }^{A} Z(t)\right\rangle\right|^{2}=|\alpha|^{2}\left\{1+e^{-\Gamma t}-2 e^{-\frac{1}{2} \Gamma t} \cos (\Delta \cdot t)\right\},
$$

with an oscillation angular frequency $|\Delta|$. The characteristic oscillation time $\tau_{\mathrm{osc}}=2 \pi|\Delta|^{-1}$ is the shortest time scale in this system. For $t \ll \tau_{\text {osc }}$, one has

$$
\left|\left\langle\left.{ }^{A}(Z-2)^{*}\right|^{A} Z(t)\right\rangle\right|^{2} \approx\left|M_{21}\right|^{2} t^{2}
$$

induced by the mass mixing.

The next shortest characteristic time in this system is the decay time $\tau_{S}=\Gamma^{-1}$, associated to the $\left|\lambda_{S}\right\rangle$ state. For $\tau_{\text {osc }} \ll t \ll \tau_{S}$, the last term in Eq.(3.1) with the interference disappears, and the two slits $\left|\lambda_{L}\right\rangle$ and $\left|\lambda_{S}\right\rangle$ contribute incoherently,

$$
\left.\left|{ }^{A}(Z-2)^{*}\right|{ }^{A} Z(t)\right\rangle\left.\right|^{2} \approx|\alpha|^{2}(2-\Gamma t) .
$$

For $t \gg \tau_{S}$, the contribution of $\left|\lambda_{S}\right\rangle$ disappears and the appearance probability simply becomes

$$
\left|\left\langle\left.{ }^{A}(Z-2)^{*}\right|^{A} Z(t)\right\rangle\right|^{2}=|\alpha|^{2} .
$$

In other words, the initially prepared $\left|{ }^{A} Z\right\rangle$ state evolves towards the stationary metastable state $\left|\lambda_{L}\right\rangle$

$$
\left|{ }^{A} Z(t)\right\rangle \rightarrow e^{-i \lambda_{L} t}\left|\lambda_{L}\right\rangle
$$

with the long lifetime $\tau_{L}=\Gamma_{L}^{-1}$ from Eq.(2.5). For a realistic time resolution $\delta t$ in an actual experiment, this regime is the interesting one, with the behavior in Eq.(3.5). The different time 
scales involved in this problem are thus

$$
\tau_{\text {osc }} \ll \tau_{S} \ll \delta t \ll t \ll \tau_{L},
$$

where $t$ refers to the elapsed time since the production of $\left|{ }^{A} Z\right\rangle$, either by nature or in the lab. Given the smallness of the mixing, the metastability of the state (3.5) is valid even for cosmological times. Therefore, for any time between the two scales $\tau_{S}$ and $\tau_{L}$, the populations of the three states involved are given by the probabilities

$$
\tau_{S} \ll t \ll \tau_{L} \Longrightarrow\left\{\begin{aligned}
P_{L}(t) & \approx 1-\Gamma_{L} t \\
P_{S}(t) & \approx 0 \\
P_{\text {g.s. }}(t) & \approx|\alpha|^{2} \Gamma t
\end{aligned}\right.
$$

where $P_{\text {g.s. }}(t)$ refers to the population of the ground state of the ${ }^{A}(Z-2)$ atom after the decay of the unstable "stationary" state $\left|\lambda_{S}\right\rangle$, with rate $\Gamma$. No matter whether $t$ refers to laboratory or cosmological times, the linear approximation in $t$ is excellent.

\section{Observables}

With this spontaneous evolution of the system, an experiment beginning its measurements a time $t_{0}$ after the ${ }^{A} Z$ was produced will probe the three-level system with relative populations $P_{L} \approx 100 \%, P_{S} \approx 0, P_{\text {g.s. }} \approx|\alpha|^{2} \Gamma t_{0}$. There are many experimental searches of candidates satisfying the resonance condition of Ref. [6]. The case of ${ }^{152} \mathrm{Gd} \rightarrow{ }^{152} \mathrm{Sm}$ mixing and decay is particularly attractive [9]. The values of the relevant parameters are the experimental $\Delta=M_{1}-M_{2}=(0.91 \pm$ $0.18) \mathrm{keV}$ for the masses of the parent " 1 " and daughter " 2 " atoms, $\Gamma=0.023 \mathrm{keV}$ for the two-hole atomic width and the $Q$-value of the ground state to ground state transition $Q=(55.70 \pm 0.18) \mathrm{keV}$. The theoretical mixing is $\left|M_{21}\right|=10^{24}\left[m_{\beta \beta} / 0.1 \mathrm{eV}\right] \mathrm{eV}$. We discover different methods, involving the third state beyond the mixed states, to be sensitive to the resonant Majorana mixing of atoms:

- Spontaneous emission from the metastable state to the daughter atom ground state. The population in the upper level $\left|\lambda_{L}\right\rangle$, as shown in Eq.(3.7a), decreases with time as $P_{L}(\Delta t) \approx$ $1-\Gamma_{L} \Delta t$, where $\Delta t=t-t_{0}$, due to the decay of the metastable "stationary" state $\left|\lambda_{L}\right\rangle$ to $\left|{ }^{A}(Z-2)_{\text {g.s. }}\right\rangle$. This process is associated to the spontaneous emission of X-rays with a rate $\Gamma_{L}$, considered in the literature after the concept of resonant mixing was introduced in Ref. [6]. For one mole of ${ }^{152} \mathrm{Gd}$, the X-ray emission rate would be of order $10^{-12} \mathrm{~s}^{-1} \sim$ $10^{-5} \mathrm{yr}^{-1}$. The initial state in the transition at observable times, being $\left|\lambda_{L}\right\rangle$, tells us that the total energy of the two-hole $\mathrm{X}$-ray radiation is displaced by $\Delta$ with respect to the characteristic $\left|{ }^{A}(Z-2)^{*}\right\rangle \rightarrow\left|{ }^{A}(Z-2)_{\text {g.s. }}\right\rangle$ X-ray spectrum, i.e. its energy release is the $Q$-value between the two atoms in their ground states (as seen in Fig. 4).

- Daughter atom population. The presence of the daughter atom in the parent ores (see Eq.(3.7c), can be probed e.g. by geochemical methods. For one mole of the nominally stable ${ }^{152} \mathrm{Gd}$ isotope produced at the time of the Earth formation we obtain an accumulated number of order $10^{4}{ }^{152} \mathrm{Sm}$ atoms. This observable could be of interest for cosmological times $t_{0}$ 
since, contrary to $\beta \beta$-decay, in the ECEC case there is no irreducible background from a $2 v$ channel for a resonant atom mixing.

We would like to emphasize that, even though $\Gamma_{L}=|\alpha|^{2} \Gamma$ ensures the probability conservation, an interpretation of Eqs.(3.7a) and (3.7c) in different terms is of interest. On the one hand, $\Gamma_{L}$ is the rate for the decay of $\left|\lambda_{L}\right\rangle$ at any time $t$, which accounts for the first observable. On the other hand, the population of the daughter atom in the ground state is obtained from the mixing probability leading to $\left|\lambda_{S}\right\rangle$ at all times, given by $|\alpha|^{2}$, times its decay rate to the ground state, $\Gamma_{S}=\Gamma$. This mixing $\times$ Decay temporal evolution explains the non-zero population of ${ }^{A}(Z-2)_{\text {g.s. }}$, producing the second observable.

- Stimulated emission from $\left|\lambda_{L}\right\rangle$. A careful reading of Eqs.(3.7) shows that the metastable state $\left|\lambda_{L}\right\rangle$ and the ground state $\left|{ }^{A}(Z-2)_{\text {g.s. }}\right\rangle$ have a natural population inversion, with an overwhelming abundance of the long-lifetime upper level of the system. This result suggests the exploitation of the bosonic properties of the X-radiation, used as a signal of the Majorana mixing in this problem, and considering the external action of an X-ray beam to stimulate the emission from the metastable level to the ground state of this atomic system.

Stimulated radiation for the emission from $\left|\lambda_{L}\right\rangle$ to the ground state $\left|{ }^{A}(Z-2)_{\text {g.s. }}\right\rangle$ could then enhance the rate and we present an estimate of the gain which could be envisaged in future facilities of X-ray beams. A setup with an incident pulsed beam allows the observation of low rate events in directions outside the beam direction and the control of background conditions in the absence of the beam. The experimental signature of the $\left.\left|\lambda_{L}\right\rangle \rightarrow||^{A}(Z-2)_{\text {g.s. }}\right\rangle$ process would be the emission of X-rays with total energy equal to the $Q$-value of the process, just like in the first observable of spontaneous emission.

For the emission between the two levels $\left|\lambda_{L}\right\rangle \rightarrow\left|{ }^{A}(Z-2)_{\text {g.s. }}\right\rangle$ of radiation with angular frequency $\omega$, stimulated radiation is described in terms of the Einstein coefficients with an induced rate $^{1}$

$$
\frac{\mathrm{d} N_{L}}{\mathrm{~d} t}=-\frac{\pi^{2} c^{3}}{\hbar \omega^{3}} \rho_{\omega} \Gamma_{L} N_{L}
$$

where $N_{L}$ is the population of the upper metastable $\left|\lambda_{L}\right\rangle$ level, $\Gamma_{L}$ its width and $\rho_{\omega}$ is the energy density of the beam per unit of angular frequency, i.e.

$$
\rho_{\omega}=\frac{\mathrm{d} E}{c \mathrm{~d} t \mathrm{~d} S \mathrm{~d} \omega}
$$

Therefore, this observable is enhanced with respect to the first one by a gain factor

$$
G=\frac{\pi^{2} c^{3}}{\hbar \omega^{3}} \rho_{\omega}
$$

which is the ratio between the stimulated and spontaneous emission rates.

In order to produce a sizable gain, one should devise a setup with as large a $\rho_{\omega}$ as possible. The transition energy of this system is of order tens of $\mathrm{keV}$, so a high-luminosity X-ray beam is

\footnotetext{
${ }^{1}$ For the sake of clarity, throughout this discussion we keep all $\hbar$ and $c$ factors.
} 
mandatory. Such high-energy beams are produced at free-electron laser (FEL) facilities, through a kind of laser consisting of very-high-speed electrons moving freely through a magnetic structure. Free-electron lasers are tunable and have the widest frequency range of any laser type, currently ranging in wavelength from microwaves, through terahertz radiation and infrared, to the visible spectrum, ultraviolet, and X-ray. The highest frequencies are obtained in XFEL facilities like the running SLAC Linac Coherent Light Source (LCLS) and the commissioned European XFEL (EXFEL) at DESY.

The determination of the gain factor one could achieve in these facilities is clearer after rewriting the spectral energy density (4.2) in terms of beam parameters,

$$
\rho_{\omega}=\frac{\hbar}{c} \frac{\mathrm{d} N}{\mathrm{~d} t \mathrm{~d} S}\left[\frac{\mathrm{d} \omega}{\omega}\right]^{-1} .
$$

Taking $\mathrm{d} N / \mathrm{d} t$ as the number of photons per pulse duration, $\mathrm{d} S$ as the beam section and $\mathrm{d} \omega / \omega$ as the full width half maximum (FWHM) spectrum width, one finds the gain factor

$$
G=\hbar(\hbar c)^{2} \frac{\pi^{2}}{(\hbar \omega)^{3}} \frac{\mathrm{d} N}{\mathrm{~d} t \mathrm{~d} S}\left[\frac{\mathrm{d} \omega}{\omega}\right]^{-1}
$$

to be written in terms of clearly defined beam properties, where $\mathrm{d} N / \mathrm{d} t \mathrm{~d} S$ is the luminosity $\mathscr{L}$ of the beam.

At EXFEL, a sound simulation of the conditions of the machine [10] gives, for typical energies of tens of $\mathrm{keV}$, the expected number of photons per pulse duration $d N / d t=10^{10} \mathrm{fs}^{-1}$ and the spectral width $\mathrm{d} \omega / \omega=1.12 \times 10^{-3}$. Nanofocusing of this X-ray FELs has been contemplated [11]; using a beam spot of the order of $100 \mathrm{~nm}$ would lead to a gain factor from (4.5) of $G \sim 100$. The continuous interaction of these $\mathrm{X}$-rays with a mole of ${ }^{152} \mathrm{Gd}$ atoms would provide a stimulated rate of order $10^{-10} \mathrm{~s}^{-1} \sim 10^{-3} \mathrm{yr}^{-1}$.

One should notice that this rate of events assumes constant irradiation of the whole target. The straightforward setup of a cylindrical target alongside the pulsating X-ray beam presents different issues. Most notoriously, pulsed beams limit the enhancing time to a fraction of the running time. EXFEL manages to produce $2.7 \times 10^{4}$ pulses per second, so that the fraction of effective time is of order $10^{-9}$; LCLS-II expects to produce pulses at $1 \mathrm{MHz}$, increasing this number by two orders of magnitude, but still far away from a promising factor. Furthermore, radiation of these energies has an attenuation length in Gd of tens of microns, limiting the amount of material one could use to a fraction of a mole. This setup has the general drawback that the high energy density effect associated with the small beam spot size is lost when considering the small interaction volume.

The attenuation of the beam is associated to its interaction with the sample, which is dominated by the photoelectric effect and, to a lesser extent, inelastic Compton scattering, leading to ionization. Successive interactions of the secondary electrons will heat the material. A recent simulation [12] of this effect under realistic experimental conditions on a cylindrical target, assuming the extreme limit that the whole absorption power is converted into heating power, shows that a temperature of about $700^{\circ} \mathrm{C}$ is reached for an incoming beam of spot size $100 \mathrm{~nm}$ and an average of $10^{14} \mathrm{X}$-ray photons/s. Since this temperature is proportional to the flux, in all high-flux ex- 
periments like the one contemplated in this work, the small-interaction-volume target is actually destroyed. The design of a macroscopic sample whith a very large number of thermally isolated micro-targets, built on a plane in transversal motion synchronized with the pulse frequency of the beam, is a subject of current interest [13]. The use of this approach in order to stimulate the $\Delta L=2$ emission rate should be explored after a suitable candidate is found. On the other hand, the limiting factors in the expected integrated rate of events also suggest an alternative ingenuity program more in the line of micro-particles inserted into a dreamed X-ray resonant cavity.

- Absorption from $\left|A(Z-2)_{\text {g.s. }}\right\rangle$. A different observable may also be considered. The existing population of the daughter atom in its ground state is, by itself, a signal of the atom Majorana mixing, as discussed before as a relic of the previous history with an initial parent atom. In addition, this population can be identified by using an intense photon beam, leading to the characteristic absorption spectrum of the daughter atom and its subsequent decay to the ground state. In the presence of a light beam, the daughter atom population would absorb those characteristic frequencies corresponding to its energy levels, which would then de-excite emitting light of the same frequency. In the case of the one mole ${ }^{152} \mathrm{Gd}$ ore that we mentioned before, all $10^{4} \mathrm{Sm}$ atoms could be easily excited to any of its $\sim 1 \mathrm{eV}$ levels using a standard pulsed laser of order $100 \mathrm{fs}$ pulse duration, with a mean power of $5 \mathrm{~W}$ and a pulse rate of $100 \mathrm{MHz}$.

Notice that these numbers imply, for a laser with FWHM spot size $\sim 40 \mu \mathrm{m}$, an absorption rate

$$
\left.\frac{\mathrm{d} N_{\mathrm{g} . \mathrm{s} .}}{\mathrm{d} t}\right|_{\text {abs }}=-60 \% N_{\mathrm{g} . \mathrm{s.}}\left[\frac{100 \mathrm{~ns}}{\tau}\right] \mathrm{fs}^{-1} .
$$

Since Sm levels have lifetimes between $10-1000$ ns [14], one expects to excite them all during the $100 \mathrm{fs}$ pulse. Disentangling the parent and daughter lines should not be difficult — the relatively small number of atomic absorption lines (compared to atomic emission lines) and their narrow width (a few pm) make spectral overlap rare, not being expected between $Z$ and $(Z-2)$ atoms.

It is worth noting from the results of this section that the bosonic nature of the atomic radiation is a property that can help in getting observable rates of the atom Majorana mixing, including the stimulated X-ray emission from the parent atom as well as the detection of the presence of the daughter atoms by means of its characteristic absorption lines. The actual values correspond to the specific case of ${ }^{152} \mathrm{Gd} \rightarrow{ }^{152} \mathrm{Sm}$, which is still off the resonance condition by at least a factor 30 , implying a factor $10^{3}$ in the rates.

\section{Conclussions}

Neutrinoless double electron capture in atoms is a quantum mixing mechanism between the neutral atoms ${ }^{A} Z$ and ${ }^{A}(Z-2)^{*}$ with two electron holes. It becomes allowed for Majorana neutrino mediation responsible of this $\Delta L=2$ transition. This Majorana mixing leads to the X-ray deexcitation of the $\left|{ }^{A}(Z-2)^{*}\right\rangle$ daughter atomic state which, under the resonance condition, has no Standard Model background from the two-neutrino decay.

The intense experimental activity looking for atomic candidates satisfying the resonance condition by means of precise measurements of atomic masses, thanks to the trapping technique, has 
already led to a few cases of remarkable enhancement effects and there is still room for additional adjustements of the resonance condition. With this situation, it is important to understand the complete time evolution of an atomic state since its inception and whether one can find, from this information, different signals of the Majorana mixing, including the possible enhancement due to the bosonic nature of atomic transition radiation.

The effective Hamiltonian for the two mixed atomic states leads to definite non-orthogonal states of mass and lifetime, each of them violating global lepton number, one being metastable with long lifetime and the other having a short lifetime. For an initial atomic state there are time periods of atom oscillations, with frequency the mass difference, and the decay of the short lived state, which are not observable for present time resolutions. For observable times, the system of the two atoms has three relevant states for discussing transitions: one highly populated state with long lifetime, one empty state with short lifetime and the ground state of the daughter atom with a small population as a result of the past history. As a consequence, this is a case of natural population inversion suggesting the possibility of stimulated radiation transitions besides the natural spontaneous X-ray emission.

The gain factor for stimulated emission due to a present radiation energy density per unit frequency has been adapted to the case of an X-ray beam in terms of conventional parameters like its luminosity, the energy and the spectrum width. Using simulated previsions of the now commissioned European XFEL facility, with a beam spot size of $100 \mathrm{~nm}$, we obtain an expected gain of 100 in the X-ray emission rate from the metastable long-lived state. This substantial gain by stimulating the X-ray emission from $\left|\lambda_{L}\right\rangle$ to $\left.\left.\right|^{A}(Z-2)_{\text {g.s. }}\right\rangle$ for interacting X-ray photons with atoms is, however, not exploited in a straightforward setup of a single pulsed beam directed towards a cylindrical target. The limiting factors of the pulse frequency and the small interaction volume are suppressing that ideal benefit for the integrated number of events.

The small population of the ground state daughter atom at observable times would be by itself a proof of the atomic Majorana mixing, given the absence of the standard two neutrino decay of the parent atom. Besides other geochemical methods, absorption rates and the subsequent emission by an intense photon beam for the daughter atom, due to its non-vanishing population of the ground state, can be clearly contemplated.

The results obtained in this work demonstrate that the knowledge of the time history of a nominally stable atom since its inception can be a source of inspiration to find appropriate observables in the search of evidence for $\Delta L=2$ double electron capture. The natural population inversion at observable times suggests stimulating the X-ray emission with gain factors which could become significant for appropriate setups. On the other hand, the presence of the daughter atom ground state can be signaled by looking for its characteristic absorption spectrum. Taking into account the ongoing searches for new isotope candidates with a better fulfillment of the resonance condition, it remains to be seen whether these processes, with the ideas on stimulating the transitions, could become actual alternatives in the quest for the Dirac/Majorana nature of neutrinos.

\section{Acknowledgments}

The authors would like to acknowledge scientific discussions with, and advice from, Massimo Altarelli, Michael Block, Albert Ferrando, Gaston Garcia and Pablo Villanueva. This research 
has been supported by MINECO Project FPA 2014-54459-P, Generalitat Valenciana Project GV PROMETEO II 2013-017 and Severo Ochoa Excellence Centre Project SEV 2014-0398. A.S. acknowledges the MECD support through the FPU14/04678 grant.

\section{References}

[1] Y. Fukuda et al. (Super-Kamiokande Collaboration), Evidence for oscillation of atmospheric neutrinos, Phys. Rev. Lett. 81 (1998) 1562 [hep-ex/9807003].

[2] Q.R. Ahmad et al. (SNO Collaboration), Direct evidence for neutrino flavor transformation from neutral current interactions in the Sudbury Neutrino Observatory, Phys. Rev. Lett. 89 (2002) 011301 [nucl-ex/0204008].

[3] T. Araki et al. (KamLAND Collaboration), Measurement of neutrino oscillation with KamLAND: evidence of spectral distortion, Phys. Rev. Lett. 94 (2005) 081801 [hep-ex/ 0406035 ].

[4] Ahn et al. (K2K Collaboration), Measurement of Neutrino Oscillation by the K2K Experiment, Phys. Rev. D74 (2006) 072003 [hep-ex/ 0606032$].$

[5] S. Pascoli, A portal to new physics, CERN Courier July-August 2016.

[6] J. Bernabeu, A. De Rujula and C. Jarlskog, Neutrinoless double electron capture as a tool to measure the electron neutrino mass, Nucl. Phys. B223 (1983) 15.

[7] S. Eliseev, C. Roux, K. Blaum, M. Block, C. Droese, F. Herfurth, H.-J. Kluge, M. I. Krivoruchenko, Yu. N. Novikov, E. Minaya Ramirez, L. Schweikhard, V. M. Shabaev, F. Åăimkovic, I. I. Tupitsyn, K. Zuber and N. A. Zubova, Resonant enhancement of neutrinoless double-electron capture in ${ }^{152} \mathrm{Gd}$, Phys. Rev. Lett. 106 (2011) 052504.

[8] V.S. Kolhinen, V.V. Elomaa, T. Eronen, J. Hakala, A. Jokinen, M. Kortelainen, J. Suhonen and J. Aysto, Accurate $Q$ value for the ${ }^{74}$ Se double-electron-capture decay, Phys. Lett. B684 (2010) 17.

[9] M. I. Krivoruchenko, F. Simkovic, D. Frekers and A. Faessler, Resonance enhancement of neutrinoless double electron capture, Nucl. Phys. A859 (2011) 140, [1012.1204 [hep-ph] ].

[10] E. Schneidmiller and M. Yurkov, DESY note, private communication from M. Altarelli.

[11] K. Yamauchi, M. Yabashi, H. Ohashi, T. Koyama and T. Ishikawa, Nanofocusing of X-ray free-electron lasers by grazing-incidence reflective optics, J. Synchrotron Rad. 22 (2015) 592, DOI:10.1107/S1600577515005093

[12] H. Wallander and J. Wallentin, Simulated sample heating from a nanofocused X-ray beam, J. Synchrotron Rad. 24 (2017) 925, DOI:10.1107/S1600577517008712

[13] P. Roedig et al., High-speed fixed-target serial virus crystallography, Nat. Meth. 14 (2017) 805, DOI:10.1038/nmeth.4335

[14] E. A. Den Hartog and J E Lawler, Radiative lifetimes of neutral samarium, J. Phys. B; At. Mol. Opt. Phys. 46 (2013) 185001, DOI:10.1088/0953-4075/46/18/185001 